\title{
Improving Healthcare Delivery with New Interactive Visualization Methods
}

\author{
Cristiana Neto ${ }^{1}$, Diana Ferreira ${ }^{1}$, António Abelha ${ }^{2}$ and José Machado ${ }^{2}$ \\ ${ }^{1}$ Informatics Department, University of Minho, Braga, Portugal \\ ${ }^{2}$ Algoritmi Research Centre, University of Minho, Guimarães, Braga, Portugal \\ $\{$ a 72064, a 72226$\}$ @alunos. uminho.pt, \\ \{abelha, jmac\} @di. uminho.pt
}

\begin{abstract}
Over the last years, the implementation and evolution of computer resources in hospital institutions has been improving both the financial and temporal efficiency of clinical processes, as well as the security in the transmission and maintenance of their data, also ensuring the reduction of clinical risk. Diagnosis, treatment and prevention of human illness are some of the most information-intensive of all intellectual tasks. Health providers often do not have or cannot find the information they need to respond quickly and appropriately to patient's medical problems. Failure to review and follow up on patient's test results in a timely manner, for example, represents a patient's safety and malpractice concern. Therefore, it was sought to identify problems in a medical exams results management system and possible ways to improve this system in order to reduce both clinical risks and hospital costs. In this sense, a new medical exams visualization platform (AIDA-MCDT) was developed, specifically in the Hospital Center of Porto (CHP), with several new functionalities in order to make this process faster, intuitive and efficient, always guaranteeing the confidentiality and protection of patients' personal data and significantly improving the usability of the system, leading to a better health care delivery.
\end{abstract}

Keywords: Information Technology, Health Information System, Electronic Health Records, Medical Exams.

\section{Introduction}

The progress of Information Technologies (IT) is an observable and unavoidable fact and currently plays a very important role in health services with the primary goal of contributing to a more efficient and high-quality health care delivery [1].

The implementation of Information Systems (IS) in hospital environments dates back to mid-1960s, given the high increase of clinical information over the years. By this time, its main functions were limited to administrative management. After 1970, larger hospitals gradually established internal information sectors [2]. Thus, the implementation of Hospital Information Systems (HIS) has improved the organization of the hospitals' large amount of information, with the purpose of not only automating, collecting and analyzing it, but also helping to support decision making.

A hospital is an institution with multiple resources that must be managed in the best way with the ultimate purposes of offering the patient a good service and optimizing 
profitability. To achieve these objectives, it is essential for each institution to have a good HIS for its current and integral management [3][4]. The most common application of those types of systems is the Electronic Health Records (EHR) system.

EHR is a computerized health system where professionals record patients' clinical information. It aims to bring together all the health care provided to a particular patient and provide a cross-sectional analysis of the patient's medical history in different services and medical units. In addition to biometric information, current prescriptions and results from imaging and laboratory tests, new and more advanced mechanisms that already integrate EHR with decision support systems begin to emerge [5][6][7].

These systems are normally distributed and heterogeneous, but the interaction among them is a crucial demand these days. In this way, the interoperability among the HIS becomes an indispensable feature in health organizations. Interoperability is the capacity of two systems to interact with each other, ensuring the understanding of the process and the exchange of data on both sides [8].

In order to solve this problem, the Agency for Integration, Diffusion and Archive (AIDA) was created and implemented in some Portuguese hospitals. AIDA is a platform developed to allow the dissemination and integration of information generated in a healthcare environment, including information on Complementary Means of Diagnosis and Therapy (MCDT) (that includes medical exams information) presented by the AIDA-VIEW platform [9][10].

Previous research has shown that health professionals often fail to review and act on test results accurately and properly. Although the reasons for the exams requests vary, accurate reporting of results is always crucial to ensure that appropriate action is taken. Several studies have also identified some practices that could improve the presentation of test results, such as highlighting the exams that still have to be visualized and the existence of groupers so related exams could be seen together. The EHR represents a significant step in the communication improvement and in the increasing of the relevant data availability and can potentially reduce communication problems associated with paper-based transmission of exams results [11][12].

Thus, to address the issue of patients' safety and quality of care, the project described in this paper emerged to help health professionals analyze and act on test results in a safe, reliable, and efficient way. The next section of this paper presents a contextualization of hospital information systems, followed by the development section where the phases of this project are explained. The section four presents the discussion of results followed by the conclusions and future work.

\section{Hospital Information Systems}

A HIS can be defined as a subsystem of a hospital with a socio-technological development, which covers all hospital information processing [9]. Its main purpose is to contribute to the quality and efficiency of healthcare. This objective is primarily oriented to the patient after being directed to health professionals as well as the functions of management and administration [9][10]. The projection and implementation of a HIS should focus on ensuring the efficient production of information in order to provide 
clinical decision-making resources. Thus, this implementation requires the existence of a management structure whose specific function focuses on the adequate allocation of resources and the definition of organizational rules [14][15]. In order to provide complete and useful resources, a HIS should also allow the extraction of clinical and management indicators as a way to improve not only decision-making, but also planning and logistics processes. EHR can be assumed as a HIS for excellence and has replaced the traditional manual recording in Paper Clinical Process (PCP). EHR may include all hospital areas with a need for registration information. Some of the advantages of EHR are to provide accurate, up-to-date and complete information about patients at the time of care and to allow quick access to patient records for more coordinated and efficient care.

\subsection{AIDA}

In order to aggregate and consolidate all the information generated by a health unit, a solid and efficient process of integration and interoperability must be developed. The main goal of interoperability in healthcare is to connect applications so data can be shared, exchanged across the healthcare environment and distributed to medical staff or patients whenever and wherever they need it [16]. This process should take into account scalability, flexibility, portability and security (confidentiality, integrity and availability) when applied in a hospital environment.

With these goals in mind, the AIDA platform (Agency for Integration, Diffusion and Archive of Medical Information) has been established in some Portuguese hospitals, including the Centro Hospitalar do Porto (CHP), which in turn connects with several systems, with the main objective of integrating, disseminating and archiving large volumes of information from several HISs [8][9][10].

AIDA is a complex system composed by simple and specialized subsystems defined as intelligent agents responsible for tasks such as the communication between heterogeneous systems, the sending and receiving of information (e.g. clinical reports, images, a set of data, prescriptions, etc.), managing and archiving of information and responding to requests properly [17][18]. AIDA's architecture is presented on Fig. 1, where it is shown the information systems integrated by AIDA: EHR; Administrative Information System (AIS); Medical Information System (MIS); and Nursing Information System (NIS) [10].

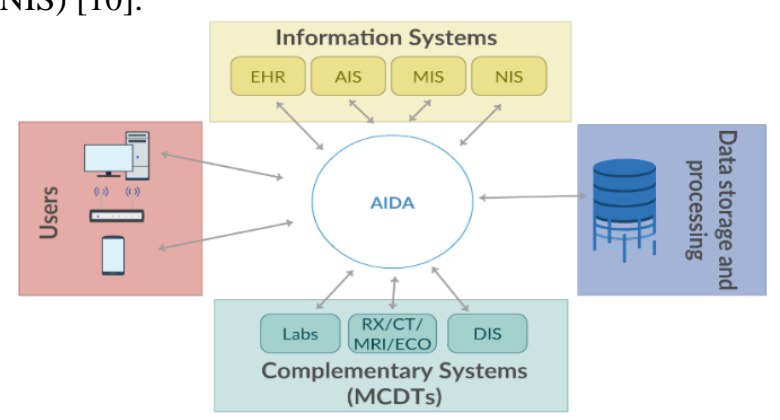

Fig. 1. AIDA platform. 
AIDA-VIEW. One other way that EHRs improve the quality of care and patient outcomes, contributing to the health of the population, is through the continuous improvement of the clinical decision making, by conducting more easily clinical trials and other studies, managing clinical knowledge, and disseminating more quickly research results to providers and patients, incorporating them rapidly into decision-support technology, and tracking the resultant changes in patient outcomes [19].

Thus, there is a general interest in minimizing the time spent in the request and consultation of MCDTs, since there is an urgent need for a more rational and efficient use of available resources, in order to minimize the time spent by the physician in these tasks and in order to ensure the provision of health care with the maximum efficiency and quality. The AIDA platform has a built-in web-based MCDTs visualization tool, the AIDA-VIEW. However, this tool is quite basic and not very efficient, presenting several negative points such as:

- Poor usability since users only have two chances of consulting the MCDTs: seeing the last 10 presented by default or consulting by specialty;

- In the visualization of the MCDTs, one cannot have the perception of exams that have already been requested, which may lead to the unnecessary realization of multiple identical exams;

- The presentation of the MCDTs is the same regardless of the user that is accessing the application and the context, which means that, in most cases, users first see information that is not relevant to them;

- There is too much information presented in the initial page that may lead to difficulties in identifying the relevant information.

Consequently, the project to reformulate this platform arises in order to solve these and other issues.

\section{Development}

The development of this project followed one of the models of software development methodology, known as SDLC, short for Software Development Life Cycle, which is widely used in several engineering and industrial fields. The model used was the waterfall model that has 5 main phases: requirements definition, design, implementation, testing, and maintenance. This was the adopted model in order to lead to the attainment of a quality product that meets the original intentions of the client [20].

\subsection{Requirements definition}

At this stage it was made an overview of the application in order to establish a basic project structure, to evaluate its feasibility and to describe the appropriate technical approaches. Here are edged the two main objectives of the software:

- Create a front-end user interface to replace the current MCDTs visualization platform; 
- Create the backend required for the front-end operation.

The identification process of the functional and non-functional requirements took several forms. Initially, the immediate and more obvious requirements to potentiate the tool were identified. Subsequently, other requirements were collected from some doctors and nurses at the CHP during numerous meetings of the PCE working group.

Thus, the main functional requirements in terms of the tool interface were:

- Creation of a hierarchical classification structure of the MCDTs, aggregating them into several groups;

- Presentation of non-visualized MCDTs and MCDTs not visualized by their specialty;

- Free text search for MCDTs;

- Filtration by module and anatomical structure;

- Presentation of the MCDTs requests already done for that patient;

- Inclusion of the MCDTs by context, that is, MCDTs related to the context at the time of access to the platform;

- Possibility to change the display settings according to user preferences;

- Preview of the report's pdf;

- Possibility of adding notes to the MCDT, working as a sort of comments section.

The requirements of the backend were limited to the ability to connect the interface to the database to manage the information necessary to operate the platform.

The main non-functional requirements collected were:

- Usability of the platform, that is, any healthcare professional using the interface must be able to easily and intuitively access the information he/she needs;

- Interoperability, since there are already several systems implemented in the hospital, this platform must be compatible with them;

- Speed, since it is a hospital environment where the less time wasted in the search for information, the better the provision of services will be;

- Security, since the platform is dealing with some confidential information.

\subsection{Design}

This stage corresponds to the process of planning the problem-solving software solution. Regarding the design of the interface, the platform was designed to have 3 modules:

- an initial module where the MCDTs are separated by the stipulated aggregators (See Fig. 2). In addition to these aggregators, it is also possible to navigate through previous structure so the transition to this new platform is made smoothly. Half of the first page is filled with the information of the patient's MCDTs requests and if an MCDT is selected, that half of the page will present the pdf preview of the MCDT report. 


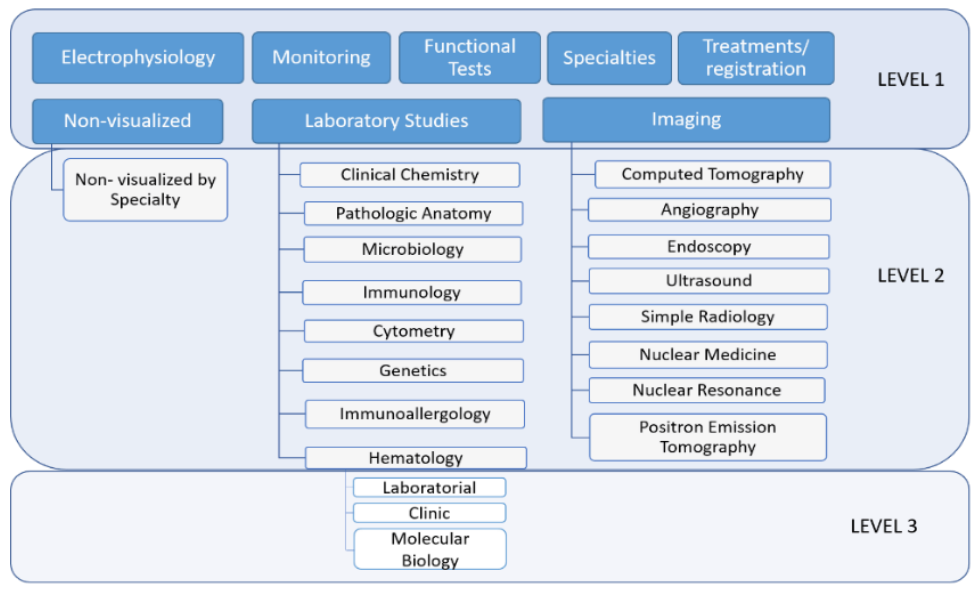

Fig. 2. Defined grouping for the exams.

- a second advanced filtering module where it is possible to perform a free text search or filter by module and anatomical location. On this page there is initially a complete list of all the MCDTs that would be filtered as the filters were selected.

- a third module with some indicators.

In terms of the backend, it was necessary to decide which hospital databases to use, what tables of those databases were needed, and which tables to add. The hospital's databases used were SIL and PCE. Within these two databases the tables to be used were summarized tables that presented information about episodes, exams, exam requests, access logs to MCDTs, user documents, among others. The tables added to the database include: a table with the groupers (which maps between the MCTD's codes and the respective grouper) and a table with the notes added to the exams.

\subsection{Implementation}

This phase is the process of converting the whole requirements and blueprints into a production environment, that is where the real code is written and compiled into an operational application [20].

In order to meet the requirements presented above, more specifically the non-functional requirements, it is very important to choose the proper technologies to be used. For the construction of the desired web application it was decided to use a JavaScript library called ReactJS. ReactJS is a JavaScript library created by Facebook and launched in 2013. This library guarantees high performance in content rendering, is easy to learn and use since it is based on building small blocks of reusable code. At the server level, ReactJS contains very fast rendering of information, which makes it very useful for quick and constant queries of the content of interest. In the backend was used PHP to make the connection between the Oracle database and the interface.

In this sense, the MCDTs visualization platform developed has an architecture based essentially on 3 components (See Fig. 3): the database where the information is stored, 
a CRUD RESTful API Web service programmed in PHP and an interface in the part of the client accessible via Web browser developed in ReactJS and using related libraries.

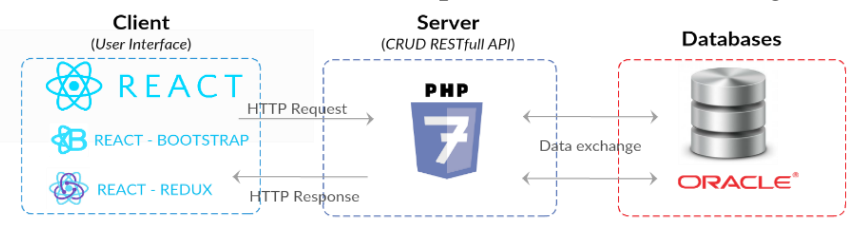

Fig. 3. Representation of the AIDA-MCDT platform's architecture.

\subsection{Testing and Maintenance}

The testing step is also known as verification and validation and is a process to check that a software solution meets the original requirements and specifications and that it accomplishes its intended purposes [20]. So, after the construction of the application itself, several tests were performed with information from different patients in different contexts. The application was also tested by several health professionals at the CHP (doctors and nurses). This process allowed errors to be corrected in order to improve the application's performance. Initially, the testing process focus the successful integration of the platform in only one service. Then, this process is applied to all the services.

Maintenance is the process of modifying a software solution after delivery and deployment to refine output, correct errors, and improve performance and quality. Additional maintenance activities can be performed in this phase including adapting software to its environment, accommodating new user requirements, and increasing software reliability [20]. This phase is now in progress since the application is being fully integrated in the hospital.

\section{Discussion of Results}

The realization of this project enabled the development of a tool to support the decision and clinical practice, namely a user-friendly computer tool for visualization of MCDTs to be implemented in the CHP named AIDA-MCDT. The main advantages of updating the architecture and the functionalities of this system were:

- Use of a modern and powerful technology, namely the ReactJS library, which presents many advantageous characteristics for system performance, namely the ability to create, reuse and combine components, a virtual DOM that results in a faster performance and a simple integration process. The use of this technology contributes to the modernization of the HIS in the CHP.

- Implementation of new modules, components and functionalities, with emphasis on the advanced filtering that ease the search for relevant information more quickly, the display of not seen exams, the presentation of MCDTs requests, the organization of MCDTs by aggregators among others.

- Introduction of context-awareness in the application. 
- Introduction of a small Business Intelligence section with only a few indicators (for example, number of patient's MCDTs per year, MCDTs performed by module and specialty and waiting time by specialty), opening doors for the development and deepening of this useful area.

- The web application is much more scalable and easier to maintain, since it presents a simple architecture and a simple process of expansion of its functionalities.

\subsection{Proof of Concept}

One of the most important steps in the design, planning, development, implementation and presentation of a prototype in IT is to perform a proof of concept since it can establish if the solution found fulfills the requirements and objectives initially defined. It also allows the identification of potential failures or errors in the proposed solution [21].

In this sense, a SWOT analysis was performed which briefly tries to define the strengths, weaknesses, opportunities and threats of the solution. Table 1 shows the results of this analysis, that is, the weaknesses, strengths, threats, and opportunities raised.

Table 1. SWOT analysis.

\begin{tabular}{|c|c|}
\hline $\begin{array}{l}\text { Strengths } \\
\text { - High scalability; } \\
\text { - High usability; } \\
\text { - Innovation: the implementation of } \\
\text { this platform enhances the moderni- } \\
\text { zation of the current system; } \\
\text { - Improvement in the decision-making } \\
\text { process. }\end{array}$ & $\begin{array}{l}\text { Weaknesses } \\
\text { - Access to the platform is only possible } \\
\text { through the internal network connec- } \\
\text { tion of the CHP; } \\
\text { - Presence of inconsistent and unneces- } \\
\text { sary information in the tables that feed } \\
\text { the platform; } \\
\text { - Due to the amount of data and the } \\
\text { complexity of some application fea- } \\
\text { tures, it may become slower. }\end{array}$ \\
\hline $\begin{array}{l}\text { Opportunities } \\
\text { - Reduction of medical errors; } \\
\text { - Achievement of better quality and } \\
\text { greater efficiency in the organization } \\
\text { through the use of new technologies; } \\
\text { - Decrease of paper usage and the con- } \\
\text { sequent increase of computerization } \\
\text { of the MCDTs. }\end{array}$ & $\begin{array}{l}\text { Threats } \\
\text { - Competition from other applications } \\
\text { in the market; } \\
\text { - Lack of interest on the part of health } \\
\text { professionals to use a new computa- } \\
\text { tional tool; } \\
\text { - Problems with network connectivity. }\end{array}$ \\
\hline
\end{tabular}

\section{$5 \quad$ Conclusion and Future Work}

This project proved to be quite viable considering the current investment in the new information technologies to improve and streamline the decision-making process, 
which is much easier when the health professionals have easy access to the information needed at the moment. It is well known that the access to the MCDTs is a crucial step in the decision-making process, and the visualization of this information as quickly as possible, as well as the reduction of information that is not visualized and left to be forgotten can lead to a substantial decrease in the occurrence of clinical errors. This project is also very useful in terms of financial management since it avoids performing repeated exams and duplicated costs, showing the user which exams have already been requested.

The work done also proved to be very challenging since the application developed can be used by several health professionals with a wide range of academic backgrounds, from doctors to nurses, to health workers and technicians in all the specialties and modules of the hospital. In this way, several requirements were raised in order to satisfy the various needs that the hospital environment generates. Accordingly, the implementation of new ITs in the hospital institution, not only meets the needs of the healthcare professionals and improves their professional activity but also improves patients' experience as they would be less exposed to eventual clinical errors.

Thus, both the new functionalities present in the application and the technologies used have contributed to the modernization of the system and to the improvement of the health care delivery. This application also potentiates the reduction of hospital costs since it prevents the execution of duplicated exams when presenting the requested tests.

Despite all of this, some improvements can still be made like, for example, the inclusion of the request for new exams in this platform and its adaptation to mobile format. Finally, the implementation and use of this application imply several security measures, so, as future work, those security measures could be enhanced to ensure the maximum security of the data used in the platform.

\section{Acknowledgments}

This work has been supported by FCT - Fundação para a Ciência e Tecnologia within the Project Scope: UID/CEC/00319/2019.

\section{References}

1. Jardim, S.V.: The electronic health record and its contribution to healthcare information systems interoperability. Procedia Technology 9, 940-948 (2013).

2. Pai, F. Y., Huang, K. I.: Applying the technology acceptance model to the introduction of healthcare information systems. Technological Forecasting and Social Change, 78(4), 650660 (2011).

3. Haux, R., Ammenwerth, E., Winter, A., \& Brigl, B.: Strategic information management in hospitals: an introduction to hospital information systems. Springer Science \& Business Media (2004).

4. Bertolini,M., Bevilacqua, M., Ciarapica, F. E., Giacchetta, G.: Business process re-engineering in healthcare management: a case study, Business Process Management Journal, 17(1), 42-66 (2011). 
5. Neto, C., Dias, I., Santos, M., Peixoto, H., \& Machado, J.: Applied Business Intelligence in Surgery Waiting Lists Management. In Healthcare Policy and Reform: Concepts, Methodologies, Tools, and Applications, 1580-1594 (2018).

6. Carter, J. H.: Electronic health records: a guide for clinicians and administrators. ACP Press (2008).

7. Salazar, M., Duarte, J., Pereira, R., Portela, F., Santos, M. F., Abelha, A., \& Machado, J.: Step towards Paper Free Hospital through Electronic Health Record. In Advances in Information Systems and Technologies, 685-694. Springer, Berlin, Heidelberg (2013).

8. Oliveira, D., Duarte, J., Abelha, A., \& Machado, J: Step towards interoperability in nursing practice. International Journal of Public Health Management and Ethics (IJPHME), 3(1), 26-37 (2018).

9. Duarte, J., Salazar, M., Quintas, C., Santos, M., Neves, J., Abelha, A., \& Machado, J: Data quality evaluation of electronic health records in the hospital admission process. In Computer and Information Science (ICIS), 2010 IEEE/ACIS 9th International Conference, 201206. IEEE (2010).

10. Duarte, J., Portela, C. F., Abelha, A., Machado, J., Santos, M. F.: Electronic health record in dermatology service. In: Cunha, M. M., Varajão, J., Powell, P., Martinho, R., editors, ENTERprise Information Systems, (156-164). Berlin, Heidelberg. Springer, Heidelberg (2011).

11. Poon, E. G., Gandhi, T. K., Sequist, T. D., Murff, H. J., Karson, A. S., \& Bates, D. W.: "I wish I had seen this test result earlier!": dissatisfaction with test result management systems in primary care. Archives of internal medicine, 164(20), 2223-2228 (2004).

12. Singh, H., Naik, A. D., Rao, R., \& Petersen, L. A.: Reducing diagnostic errors through effective communication: harnessing the power of information technology. Journal of General Internal Medicine, 23(4), 489-494 (2008).

13. Poon, E. G., Wang, S. J., Gandhi, T. K., Bates, D. W., \& Kuperman, G. J: Design and implementation of a comprehensive outpatient results manager. Journal of Biomedical Informatics, 36(1), 80-91 (2003).

14. Duarte, J., Castro, S., Santos, M., Abelha, A., \& Machado, J. M.: Improving quality of electronic health records with SNOMED. Procedia technology, 16, 1342-1350 (2014).

15. Lippeveld, T., Sauerborn, R., \& Bodart, C. (Eds.): Design and implementation of health information systems, 281. Geneva: World Health Organization (2000).

16. Miranda, M., Duarte, J., Abelha, A., Machado, J. M., \& Neves, J.: Interoperability and healthcare (2009).

17. Pontes, G., Portela, C. F., Rodrigues, R., Santos, M. F., Neves, J., Abelha, A., \& Machado, J.: Modeling Intelligent Agents to Integrate a Patient Monitoring System. In: Trends in Practical Applications of Agents and Multiagent Systems, 139-146. Springer, Cham (2013).

18. Cardoso, L., Marins, F., Portela, F., Santos, M., Abelha, A., \& Machado, J.: A multi-agent platform for hospital interoperability. In Ambient Intelligence-Software and Applications, 127-134. Springer, Cham (2014).

19. G. Goldschmidt, P.: HIT and MIS: Implications of health information technology and medical information systems. Commun, ACM, 48, 68-74 (2005).

20. Bassil, Y.: A simulation model for the waterfall software development life cycle (2012).

21. Pereira, R., Salazar, M., Abelha, A., \& Machado, J.: SWOT Analysis of a Portuguese Electronic Health Record. In: Conference on e-Business, e-Services and e-Society, 169-177. Springer, Berlin, Heidelberg (2013). 